\title{
Role of Anterolateral Thigh Flaps in Distal Lower Extremity Reconstruction: Assessment of Functionality, Aesthetics, and Patient Satisfaction
}

\author{
Michael Trostler, MS, MD ${ }^{1}$ Johnathan Shih, BS² Gabriel M. Klein, MD ${ }^{3} \quad$ Alexander B. Dagum, MD ${ }^{1}$ \\ Duc T. Bui, MD ${ }^{1}$ Mark A. Gelfand, MD ${ }^{1}$ \\ ${ }^{1}$ Division of Plastic and Reconstructive Surgery, Stony Brook \\ Medicine, Stony Brook, New York \\ 2 Stony Brook University School of Medicine, Stony Brook, New York \\ 3 Department of General Surgery, Stony Brook Medicine, Stony Brook,

\begin{abstract}
Address for correspondence Duc T. Bui, MD, Division of Plastic and Reconstructive Surgery, Stony Brook Medicine, 101 Nicolls Rd, HSC 19-060, Stony Brook, NY 11794
\end{abstract} \\ (e-mail: Duc.Bui@stonybrookmedicine.edu).
}

New York

J Reconstr Microsurg Open 2017;2:e83-e89.

\begin{abstract}
Background Distal lower extremity reconstruction presents many challenges due to the suboptimal vascular supply, the weight-bearing necessity, movement, and multiple tissue types that can be easily damaged by trauma, from bones, tendons, ligaments, muscle, and soft tissue loss. The anterolateral thigh flap provides a reconstructive option for large defects with good coverage and minimal donor-site morbidity.

Methods After a chart review of all anterolateral thigh flaps, the patients were contacted to take a survey regarding their satisfaction and functional ability and to meet for measurements of their feet after debulking procedures.

Results A total of 13 patients were included in the study, with traumatic injuries comprising $84 \%$ of cases. There was a significant difference in sizes of the midfoot (mean: 10.27 vs. 9.53, $p=0.004$ ) and the hindfoot (mean: 12.92 vs. $12.2, p=0.023$ ) when comparing the affected side to the unaffected side. The majority, $77 \%$, of our patients underwent debulking procedures. Overall, 75\% of traumatic injury patients had $>90 \%$ maximum functionality score. All posttraumatic reconstruction results

Keywords

- anterolateral thigh flap

- soft tissue reconstruction

- lower extremity were rated satisfied to very satisfied by the patients. All posttraumatic patients were able to walk after their procedures, although three patients did require custom-made shoes with inserts or braces.

Conclusion The anterolateral thigh flap is a versatile reconstructive option that can be used in the distal lower extremity and is especially useful after a traumatic injury as it allows for functional recovery and high patient satisfaction after debulking.
\end{abstract}

Free flap reconstruction of soft tissue defects in the distal lower extremity presents inherent challenges given the suboptimal vascularity of the distal limb, and continuous weight-bearing forces, movement, and friction. Successful repair in this anatomic region is even more difficult in the posttraumatic patient, as free tissue transfers must contend with additional constraints of possible concomitant bone and ligamentous injury, vascular compromise, and received

November 1, 2016 accepted after revision May 10, 2017
DOI https://doi.org/

10.1055/s-0037-1603941. ISSN 2377-0813.
Copyright $\odot 2017$ by Thieme Medical Publishers, Inc., 333 Seventh Avenue, New York, NY 10001, USA. Tel: +1(212) 584-4662.
License terms

(ㄷ) (i) $\ominus$ (5) 
extensive soft tissue injuries that extend beyond the immediate wound. Although muscle and musculocutaneous flaps are taken from latissimus dorsi or rectus abdominous donor sites have traditionally been utilized for coverage of such defects, in recent times there has been increasing evidence to support the use of the free fasciocutaneous anterolateral thigh (ALT) flap in posttraumatic reconstruction of the distal lower limb. ${ }^{1-4}$ Many features of the ALT flap make it ideal for soft tissue repair in the distal lower limb, including its large skin territory, long, and robust vascular pedicle, the potential for sensory preservation, and minimal donor-site morbidity. ${ }^{1}$ First described by Song et al as a novel free flap concept for head and neck reconstruction, the ALT flap has since established itself as an excellent choice for primary coverage of traumatic soft tissue defects in the lower extremity. ${ }^{5-7}$ Song et al first described a possible area of $800 \mathrm{~cm}^{2}$, with a pedicle that ranged from 10 to $12 \mathrm{~cm}$, although pedicles have been reported up to $16 \mathrm{~cm}$, and a thickness that varied along its length from 1 to $3 \mathrm{~cm} .{ }^{5-8}$ The ALT is a versatile flap which can cover a variety of anatomic sites including the scalp, head and neck, trunk, and lower extremity with a low rate of dehiscence (3.8\%), hematoma/seroma (1-2\%), and skin grafting for the donor site(14.5\%), it is an aesthetic choice with a scar easily hidden by wearing pants or shorts. ${ }^{5,9}$

In this study, we reviewed our use of the free ALT flap for posttraumatic reconstruction of complex soft tissue defects in the lower extremity. Specifically, we evaluated flap outcomes and patient satisfaction from a functional and aesthetic standpoint. There has been a paucity of research focusing on patient-centered outcomes as shown in a review by Economides et $\mathrm{al}^{10}$ and we aimed to remedy this in our patient population.

\section{Methods}

This was a retrospective chart review of all ALT flaps done from 2010 to 2014 after the chart review was completed, we then contacted the patients. A total of 14 patients were found to have undergone an ALT flap to the lower extremity. Patient charts were reviewed for background information regarding the nature of the initial injury, specifics regarding location and size of the defect, initial, and secondary procedures performed, the requirement for skin grafting and postoperative complications. When the patients were contacted, they were asked if they would participate in a survey about their functional and aesthetic outcomes and if they would be willing to have a member of the research team meet them and take measurements of their lower extremities to compare the affected side to the unaffected side. The survey included the ability to wear shoes, subjective comparison to opposite side, pain, wounds, the ability to ambulation, activity level, and patient satisfaction level. Five of the six patients who were measured consented to do the Lower Extremity Function Scale (LEFS) to measure their functional limitations. ${ }^{11}$ Statistical analysis was performed with SPSS v22 (IBM Corp.) and included descriptive analysis and $t$-test, with significance set at $p<0.05$.
Table 1 Demographics

\begin{tabular}{|c|c|}
\hline & $N(\%)$ \\
\hline Age (y) & 42.07 (range: 9-78) \\
\hline \multicolumn{2}{|l|}{ Gender } \\
\hline Male & $12(92.3)$ \\
\hline Female & $1(7.7)$ \\
\hline \multicolumn{2}{|l|}{ Cause of injury } \\
\hline Trauma & $11(84.6)$ \\
\hline Necrotizing fasciitis & $1(7.7)$ \\
\hline Nonhealing ulcer & $1(7.7)$ \\
\hline
\end{tabular}

\section{Results}

A total of 13 patients were included in this study with demographics shown in - Table 1. Out of these 13, 11 underwent flap reconstruction secondary to traumatic injury, while the remaining 2 underwent reconstruction for necrotizing fasciitis and a nonhealing diabetic ulcer, individual characteristics seen in - Table 2 (-Figs. 1-4). The causes of the traumatic injuries varied, including motor vehicle collisions, motorcycle accidents, and injuries related to lawn mowers and workplace hazards. The nature of these injuries included crush, avulsion, degloving, dislocation of joints, fractures, lacerations, amputations, compartment syndrome, and ligamentous injury. The defects that were covered ranged in size from 50 to $350 \mathrm{~cm}^{2}$ with a mean of $128.8 \mathrm{~cm}^{2}$. Overall, $46 \%$ of patients required additional coverage with a split-thickness skin graft, and $7.7 \%$ required a full-thickness skin graft due to the extensive and multifocal nature of their traumatic injuries. Six patients consented to participate in a postoperative survey, with five patients completing the LEFS. We found that three patients reported $>90 \%$ of maximal functionality while the other two reported a 45 to $50 \%$ functional score. Patients reporting lower functional outcomes included the only nontraumatic injury patient who presented with a chronic nonhealing foot ulcer and a patient who suffered severe traumatic crush injury with degloving and a partial amputation of the foot. It should be noted that the patient who suffered the severe crush injury was very satisfied with the results, had minimal pain, and was able to ambulate comfortably with a brace. The patient with the chronic ulcer was the only patient who was unsatisfied with his result due to the increased size of his affected foot with the added bulk kept to prevent recurrence of the chronic ulcer over a bony prominence. He can walk only with a walker and also uses a wheelchair, but has no pain, and has had no ulcers. Ten of the patients included in this series had at least one debulking procedure including five of the six patients that were able to be contacted, and these five patients were satisfied or very satisfied.

None of the patients surveyed had ulcers or chronic pain. Only one patient was unable to wear shoes due to the size of the flap, while the remaining patients were able to wear 


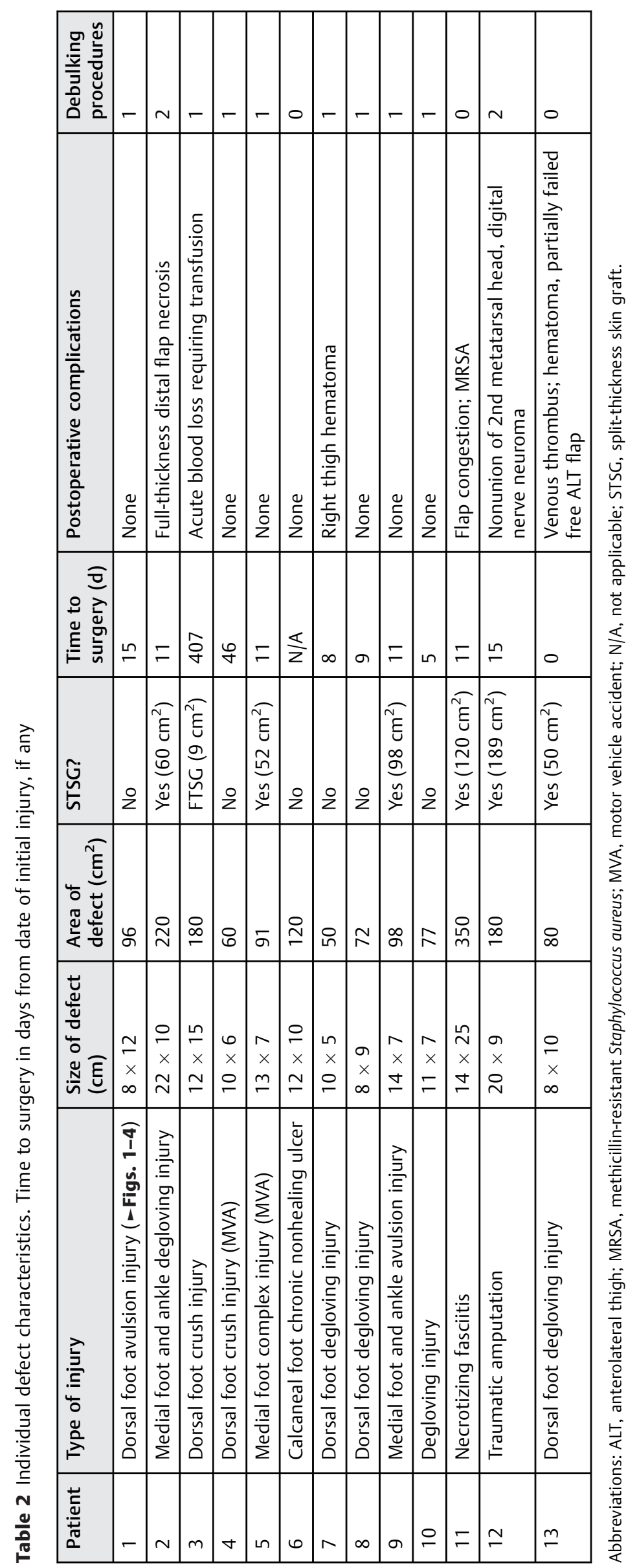




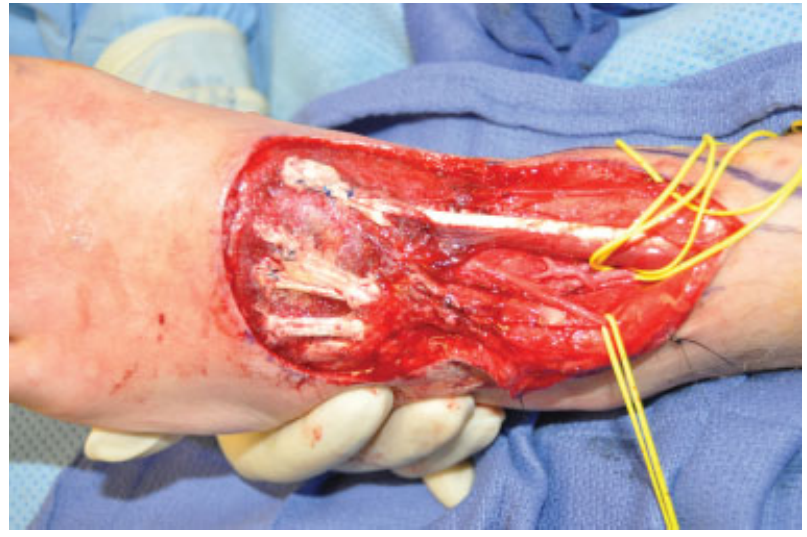

Fig. 1 Patient 1 intraoperative exposure after left dorsal foot crush and avulsion injury from golf cart accident.

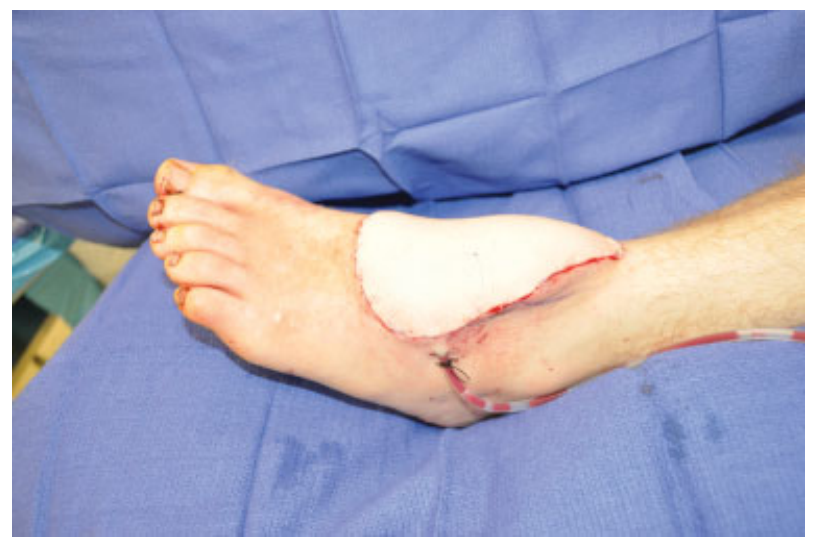

Fig. 2 Patient 1 immediate postoperative image after ALT flap. ALT, anterolateral thigh.

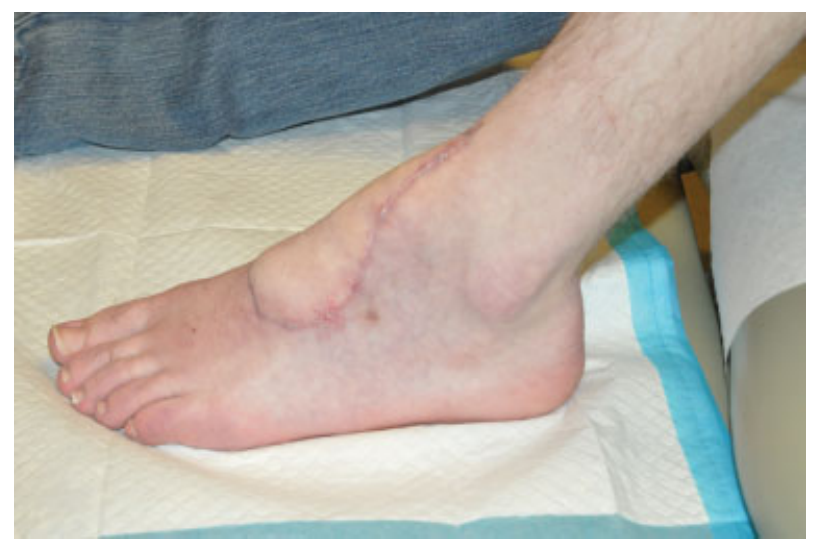

Fig. 3 Patient 1, 2 months after debulking procedure.

shoes comfortably, with three patients requiring custommade shoes with inserts ( - Table 3 ).

Six patients were able to be contacted and consented to have measurements of bilateral lower extremities taken (-Table 4). With the hypothesis that the surgical limb with flap inset would be significantly larger than the nonsurgical limb, it was found that there was a statistically significant difference in both the midfoot (mean: 10.27 vs. 9.53, $p=0.004$ ) and the hindfoot (mean: 12.92 vs. 12.2,

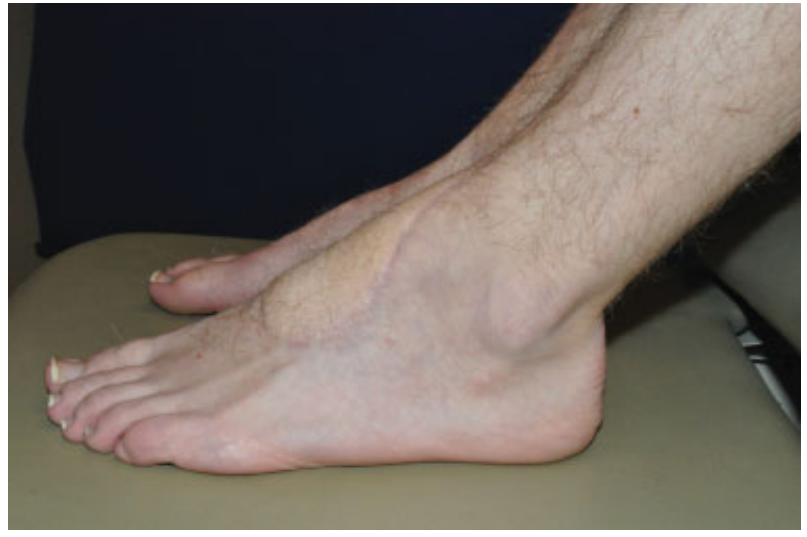

Fig. 4 Patient 1, 1.5 years after debulking. The patient is able to ambulate and wear regular shoes without limitation or activity.

$p=0.023)$. There was no statistical difference between the forefoot (mean: 9.04 vs. $8.98, p=0.45$ ) and the ankle (mean: 10.75 vs. $10.42, p=0.25$ ). Similar results were obtained when we evaluated only patients who underwent reconstruction secondary to traumatic injury, with a statistically significant difference in both midfoot $(p=0.009)$ and hindfoot $(p=0.03)$ measurements.

\section{Discussion}

Reconstruction of soft tissue defects in the distal lower extremity remains challenging, particularly in the posttraumatic setting. In the literature, the fasciocutaneous ALT flap has established itself as a versatile alternative to the traditional musculocutaneous flap for the repair of such defects, with the additional benefit of functional muscle unit preservation at the donor site. The ALT flap offers an expansive territory of coverage secondary to robust perfusion from perforator arteries that arise from the descending branch of the lateral femoral circumflex artery. Koshima et al reported a potential surface area of $25 \times 18 \mathrm{~cm}$ in the nonthinned flap, while others have more recently described success with flaps of up to $35 \mathrm{~cm} \times 15 \mathrm{~cm} .^{1,3,7}$ The long vascular pedicle of the ALT flap ensures flap viability in the traumatic foot by allowing for microvascular anastomosis to a healthy recipient vessel located sufficiently far away from the site of injury. In a patient who has suffered an injury to his foot, the intrinsic thickness of the subcutaneous fat layer in the ALT flap is helpful and sometimes requisite for primary reconstruction and stabilization. In the postoperative phase, however, excess thickness of the ALT flap is a common source of dissatisfaction among patients, as it may interfere with normal functionality, ability to wear shoes and achievement of satisfactory cosmesis. One solution to minimize such potential complications is to perform primary thinning of the ALT flap during initial reconstruction. Kimura et al and others have reported success with 3 to $4 \mathrm{~mm}$ flaps that can span up to $13 \mathrm{~cm}$ in radius from the point at which the perforating vessel enters the skin. ${ }^{1,12,13}$ In Western countries ALT flaps are on average considerably thicker; excessive thinning has been associated with increased flap 

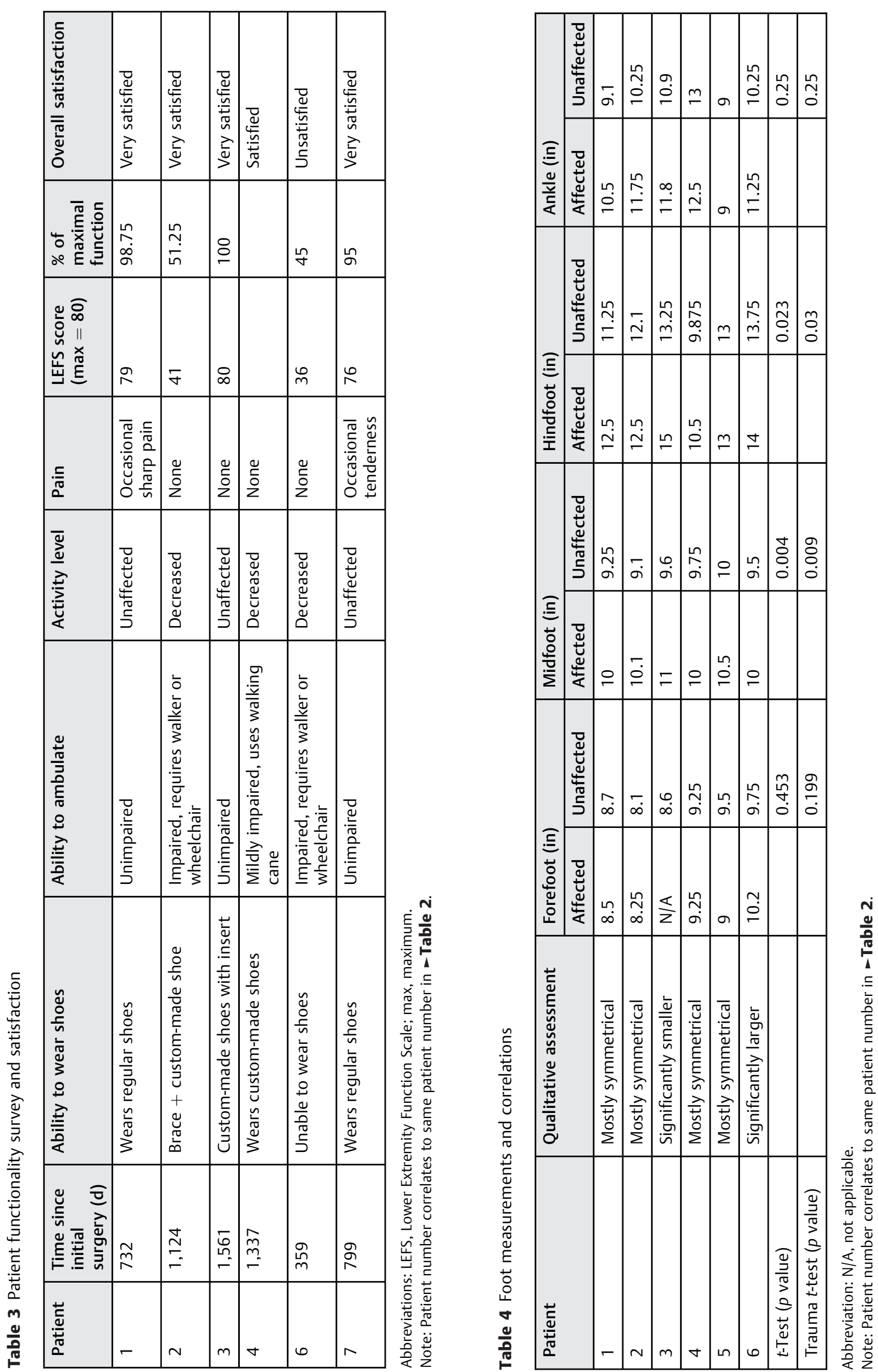
morbidity. ${ }^{14}$ Consequently, in the setting of traumatic injury, surgical debulking is done at a later time. In our study, $77 \%$ of patients underwent at least one debulking procedure. While there remained a statistically significant difference in foot size among these patients, all posttraumatic patients who underwent secondary debulking procedures were satisfied with the results. In the cases presented, the only patient who reported dissatisfaction was the patient who received ALT flap coverage for a chronic diabetic ulcer. This patient was not offered a secondary debulking procedure, as it was believed that the patient would require the extra flap thickness to prevent ulcer recurrence. Liposuction has also been supported in the literature as a less invasive alternative to surgical debulking for secondary thinning and contouring of the ALT flap. ${ }^{15,16}$

The ALT flap is an important tool for soft tissue reconstruction of the distal lower extremity in the posttraumatic setting. The primary reconstruction requires a robust, wellperfused flap with maximal healing potential. After success with the immediate reconstruction, our focus then shifts toward optimization of the functional and aesthetic outcome. The ALT flap has excellent debulking potential; this is crucial in the lower extremity, where the ability to ambulate and wear normal shoes is highly dependent on the size of the flap and the mobility of the underlying joints and tendons. All of our posttraumatic patients that were able to be surveyed were satisfied to greatly satisfied with their results after debulking procedures were finished, and three of the four patients who completed the LEFS survey had $\geq 95 \%$ functionality. Secondary reconstructive procedures are often necessary and need to be part of the algorithm of lower extremity salvage. ${ }^{17}$ However revisions and debulking surgeries are not just aesthetic but are functional as means to improve range of motion and ability to wear normal shoes in cases of foot and ankle reconstruction. Recent trends have been to elevate the ALT flap at the suprafacial or suprascarpal level to provide a thinner flap, which allows for fewer debulking procedures, and has been shown to have good outcomes by Seth and Iorio ${ }^{18}$ who looked at a series of 25 patients with flaps placed all over the body. The superthin ALT is an acceptable technique with strong outcome if there is time to plan the surgery and the patient is stable, but in our series many of our trauma patients had exposed bone and hardware from concomitant or recent orthopedic procedures there was the need to take a musculocutaneous ALT flap which would preclude the use of the superthin ALT or individual factors from these multitrauma patients lead us to a thicker flap with a shorter operative time with the plan to debulk the flap when the patient is recovered. A fullthickness ALT allows for easy debulking and a high satisfaction rating among our surveyed patients.

The weaknesses of this study are the small number of patients and our inability to follow-up with several patients. Several patients in the study could not be contacted as they were illegal immigrants without physical addresses, working telephone numbers, or any listed means of communicating with them, and one patient who was contacted declined to participate.

\section{Conclusion}

In our experience, the free ALT flap is a versatile option for free tissue transfer that may be safely considered in the reconstruction of large and complex traumatic soft tissue defects of the distal lower extremity. In addition to its reconstructive potential, it has excellent functional and aesthetic results which may be achieved through secondary surgical debulking.

\section{References}

1 Yildirim S, Gideroğlu K, Aköz T. Anterolateral thigh flap: ideal free flap choice for lower extremity soft-tissue reconstruction. J Reconstr Microsurg 2003;19(04):225-233

2 Demirtas Y, Kelahmetoglu O, Cifci M, Tayfur V, Demir A, Guneren E. Comparison of free anterolateral thigh flaps and free musclemusculocutaneous flaps in soft tissue reconstruction of lower extremity. Microsurgery 2010;30(01):24-31

3 Park JE, Rodriguez ED, Bluebond-Langer R, et al. The anterolateral thigh flap is highly effective for reconstruction of complex lower extremity trauma. J Trauma 2007;62(01):162-165

4 Ozkan O, Coşkunfirat OK, Ozgentaş HE. The use of free anterolateral thigh flap for reconstructing soft tissue defects of the lower extremities. Ann Plast Surg 2004;53(05):455-461

5 Nasajpour H, Steele MH. Anterolateral thigh free flap for "head-totoe" reconstruction. Ann Plast Surg 2011;66(05):530-533

6 Song YG, Chen GZ, Song YL. The free thigh flap: a new free flap concept based on the septocutaneous artery. Br J Plast Surg 1984; 37(02):149-159

7 Koshima I, Fukuda H, Yamamoto H, Moriguchi T, Soeda S, Ohta S. Free anterolateral thigh flaps for reconstruction of head and neck defects. Plast Reconstr Surg 1993;92(03):421-428, discussion 429-430

8 Horn D, Jonas R, Engel M, Freier K, Hoffmann J, Freudlsperger C. A comparison of free anterolateral thigh and latissimus dorsi flaps in soft tissue reconstruction of extensive defects in the head and neck region. J Craniomaxillofac Surg 2014;42(08):1551-1556

9 Lakhiani C, DeFazio MV, Han K, Falola R, Evans K. Donor- site morbidity following free tissue harvest from the thigh: a systematic review and pooled analysis of complications. J Reconstr Microsurg 2016;32(05):342-357

10 Economides JM, Patel KM, Evans KK, Marshall E, Attinger CE. Systematic review of patient-centered outcomes following lower extremity flap reconstruction in comorbid patients. J Reconstr Microsurg 2013;29(05):307-316

11 Binkley JM, Stratford PW, Lott SA, Riddle DL; North American Orthopaedic Rehabilitation Research Network. The Lower Extremity Functional Scale (LEFS): scale development, measurement properties, and clinical application. Phys Ther 1999;79(04): 371-383

12 Kimura N, Satoh K. Consideration of a thin flap as an entity and clinical applications of the thin anterolateral thigh flap. Plast Reconstr Surg 1996;97(05):985-992

13 Kimura N, Satoh K, Hasumi T, Ostuka T. Clinical application of the free thin anterolateral thigh flap in 31 consecutive patients. Plast Reconstr Surg 2001;108(05):1197-1208, discussion 1209-1210

$14 \mathrm{Yu}$ P. Characteristics of the anterolateral thigh flap in a Western population and its application in head and neck reconstruction. Head Neck 2004;26(09):759-769

15 Askouni EP, Topping A, Ball S, Hettiaratchy S, Nanchahal J, Jain A. Outcomes of anterolateral thigh free flap thinning using liposuction following lower limb trauma. J Plast Reconstr Aesthet Surg 2012;65(04):474-481 
16 Duffy FJ Jr, Brodsky JW, Royer CT. Preliminary experience with perforator flaps in reconstruction of soft-tissue defects of the foot and ankle. Foot Ankle Int 2005;26(03):191-197

17 Nelson JA, Fischer JP, Haddock NT, et al. Striving for normalcy after lower extremity reconstruction with free tissue: the role of secondary esthetic refinements. J Reconstr Microsurg 2016; 32(02):101-108

18 Seth AK, Iorio ML. Super-thin and suprafascial anterolateral thigh perforator flaps for extremity reconstruction. J Reconstr Microsurg 2017. Doi: 10.1055/s-0037-1601422 